\title{
Clinical Research and Development
}

National Cancer Institute

\section{Source}

National Cancer Institute. Clinical Research and Development. NCI Thesaurus. Code C142435.

The development and testing of a drug, device, or therapy in human subjects to determine safety and efficacy. 